\title{
Chiral Expansion, Renormalization and the Nuclear Force
}

\author{
E. Ruiz Arriola ${ }^{\text {a }}$ and M. Pavón Valderrama ${ }^{\mathrm{a}}$
}

${ }^{a}$ Departamento de Física Atómica, Molecular y Nuclear (Granada) Spain

The renormalization of singular chiral potentials as applied to NN scattering and the structure of the deuteron is discussed. It is shown how zero range theories may be implemented non-perturbatively as constrained from known long range NN forces.

\section{Introduction}

A piece of standard wisdom in nuclear physics has been the long distance dominance of pion exchanges of the nuclear force while the short range component is fairly unknown [ 1]. Such a situation seems a fertile ground for Effective Field Theories [ 2, 3]. Actually, the functional dependence of the long range piece is calculable within chiral perturbation theory [4, 5] yielding for the reduced potential $(U(r)=M V(r))$

$U(r)=M_{N} m_{\pi}\left\{\frac{m_{\pi}^{2}}{f_{\pi}^{2}} W^{(1)}\left(m_{\pi} r\right)+\frac{m_{\pi}^{4}}{f_{\pi}^{4}} W^{(2)}\left(m_{\pi} r\right)+\frac{m_{\pi}^{4}}{f_{\pi}^{4}} \frac{m_{\pi}}{M_{N}} W^{(3)}\left(m_{\pi} r\right)+\ldots\right\} \quad(r \neq 0)$

with $M_{N}$ the nucleon mass and $m_{\pi}$ the pion mass and $f_{\pi}$ the pion weak decay constant. Based on these or similar chiral potentials [ 6, 7] successful description of low energy scattering data [1] is achieved. However, one of the problems which immediately arises refers to the location of a sensible boundary between short and long distances such that model independence is ensured. The most obvious candidate for a short distance cut-off is the smallest probing wavelength, which in the case of elastic NN interaction corresponds to $\lambda=0.5 \mathrm{fm}$. Thus, to describe satisfactorily elastic NN scattering from threshold up to the pion production threshold a fairly good knowledge of the force above $0.5 \mathrm{fm}$ is needed. The crucial question is whether such a statement can be verified regardless on the uncertainties at short distances. Any effective description of long wavelength phenomena requires that distances shorter than the probing wavelength become irrelevant, and although the statement is quite obvious as a physical requirement the corresponding mathematical implementation is far from trivial. The local character of Eq. (11) suggests using coordinate space methods which, due to the boundary value character of the Schrödinger equation have the obvious advantage of a rather neat separation between disjoint regions of space which can be connected at the boundaries. This property does not hold in momentum space, where all scales are intertwined. In addition, a clear advantage of this coordinate space analysis is the hierarchy of equations that arises in the renormalization problem [ 8, 9].

${ }^{*}$ Speaker at International IUPAP Conference on Few-Body Problems in Physics (FB18), Santos-Sao Paulo (Brasil), 21-26 August 2006. Work supported by DGI and FEDER funds, under contract FIS2004- and by the Junta de Andalucía grant no. FM-225 and EURIDICE grant number HPRN-CT-2003-00311. 


\section{The singlet ${ }^{1} S_{0}$ channel}

Our points are best illustrated for s-waves $\left({ }^{1} S_{0}\right)$ where the Schrödinger equation reads

$$
-u_{k}^{\prime \prime}(r)+U(r) u_{k}(r)=k^{2} u_{k}(r)
$$

The asymptotic solution for finite energy is normalized as

$u_{k}(r) \rightarrow \frac{\sin \left(k r+\delta_{0}\right)}{\sin \delta_{0}} \quad(r \rightarrow \infty)$

In the zero energy limit (using $\delta_{0}(k) \rightarrow-\alpha_{0} k+\ldots$ ) one gets

$u_{0}(r) \rightarrow 1-\frac{r}{\alpha_{0}} \quad(r \rightarrow \infty)$

Orthogonality of solutions $0 \leq r \leq \infty$ implies that

$\delta(k-p)=N \int_{0}^{\infty} u_{k}(r) u_{p}(r) d r$

with $N$ a suitable normalization constant. Straightforward manipulation yields

$0=u_{k}^{\prime} u_{p}-\left.u_{k} u_{p}^{\prime}\right|_{0^{+}}$

which implies an energy independent boundary condition. In general a short distance limit $r_{c} \rightarrow 0^{+}$may be required. The relation to a sharp cut-off in momentum space is $r_{c}=\pi / 2 \Lambda[$ [10]. From a mathematical viewpoint this is equivalent to looking for selfadjoint extensions of hermitian operators on the Hilbert space with a common domain within which completeness of solutions is ensured. This condition can be deduced from the smallness of probability at small distances [11].

In the absence of a potential, $U(r)=0$, (pionless theory), Eqs. (3) and (41) become the solution everywhere and hence taking the limit $p \rightarrow 0$ one has

$k \cot \delta_{0}(k)=\frac{u_{k}^{\prime}(0)}{u_{k}(0)}=\frac{u_{0}^{\prime}(0)}{u_{0}(0)}=-\frac{1}{\alpha_{0}}$

This is the effective range expansion $k \cot \delta_{0}=-\frac{1}{\alpha_{0}}+\frac{1}{2} r_{0} k^{2}+v_{2} k^{4}+\ldots$ with $r_{0}=v_{2}=$ $\ldots=0$. The vanishing $r_{0}$ is a sufficient condition for causality, $r_{0} \leq 0$ [ [12]. Under a weak potential perturbation one has, after renormalization, the result

$k \cot \delta_{0}(k)=-\frac{1}{\alpha_{0}}+\int_{0}^{\infty} d r U(r)\left(\left[\cos (k r)-\frac{\sin (k r)}{\alpha_{0} k}\right]^{2}-\left[1-\frac{r}{\alpha_{0}}\right]^{2}\right)+\ldots$

The renormalized effective range is entirely predicted from the potential at all distances

$r_{0}=4 \int_{0}^{\infty} d r r^{2} U(r)\left(1-\frac{r}{\alpha_{0}}\right)^{2}+\ldots$

Note the extra power suppression at the origin when $\alpha_{0}$ is fixed, indicating short distances become less important. Finite cut-off approaches not only fix a short distance finite cutoff in the lower limit $r_{c}$ but also add an extra short distance contribution $r_{0, S}$. Also, if 

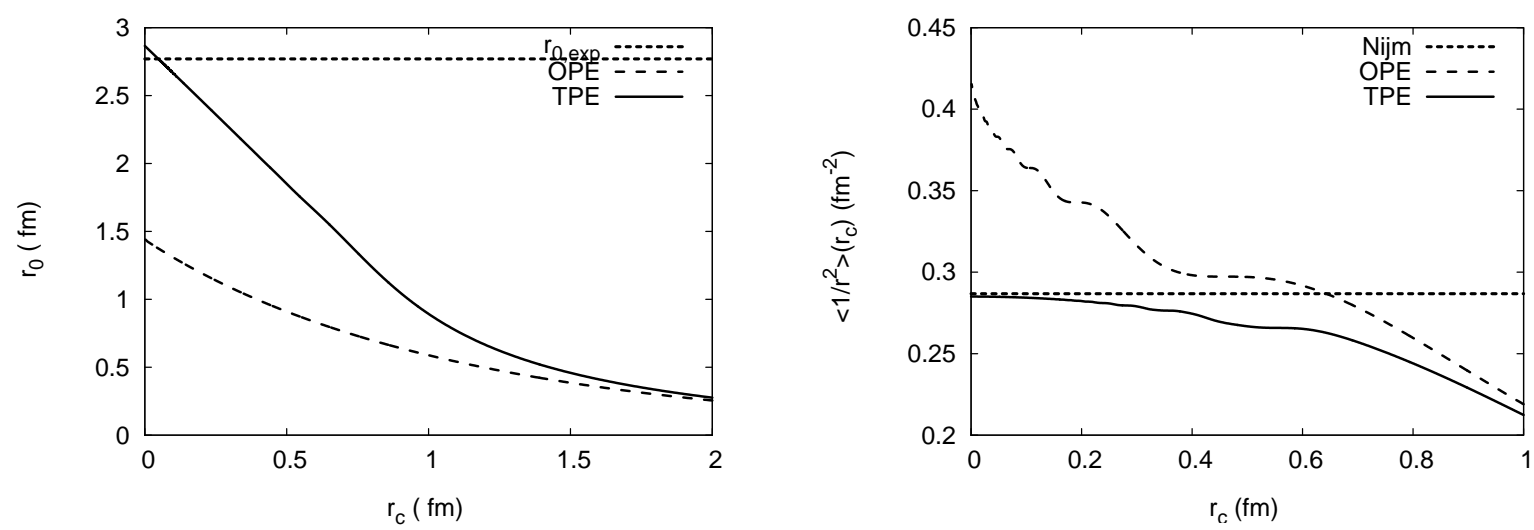

Figure 1. Cut-off dependence of $\mathrm{OPE}_{\mathrm{LO}}$ and $\mathrm{TPE}_{\mathrm{N} 2 \mathrm{LO}}$ observables compared to the Nijm II and Reid 93 potential results. Left: ${ }^{1} S_{0}$ Effective range. Right: Deuteron $\left\langle r^{-2}\right\rangle$ moment.

Table 1

${ }^{1} S_{0}$ threshold parameters, $k \cot \delta_{0}=-\frac{1}{\alpha_{0}}+\frac{1}{2} r_{0} k^{2}+v_{2} k^{4}+\ldots$, for fully iterated potentials (f.i.) and for perturbation theory based on OPE distorted waves [11]. NijmII from [1].

\begin{tabular}{|c|ccc|c|c|ccc|}
\hline${ }^{1} S_{0}$ & LO $_{\text {f.i. }}$ & NLO $_{\text {f.i. }}$ & NNLO $_{\text {f.i. }}$ & Exp. & Nijm II & LO $_{\text {pert }}$ & NLO $_{\text {pert }}$ & $\mathrm{N}^{2}$ LO $_{\text {pert }}$ \\
\hline$\alpha_{0}(\mathrm{fm})$ & Input & Input & Input & $-23.74(2)$ & -23.73 & Input & Input & Input \\
$r_{0}(\mathrm{fm})$ & 1.44 & 2.29 & 2.86 & $2.77(5)$ & 2.67 & 1.383 & Input & Input \\
$v_{2}\left(\mathrm{fm}^{3}\right)$ & -2.11 & -1.02 & -0.36 & - & -0.48 & -2.053 & Input & Input \\
$v_{3}\left(\mathrm{fm}^{5}\right)$ & 9.48 & 6.09 & 4.86 & - & 3.96 & 9.484 & Input & Input \\
$v_{4}\left(\mathrm{fm}^{7}\right)$ & -51.31 & -35.16 & -27.64 & - & -19.88 & -50.74 & -61.19 & -64.07 \\
\hline
\end{tabular}

$r_{0, S} \neq 0$ for $r_{c}=0$ there is no additional power suppression of the potential at the origin, which is counterintuitive. Orthogonality implies that $r_{0, S} \rightarrow 0$ as $r_{c} \rightarrow 0$, and similarly with the short distance components of $v_{2}$, etc. The question is whether for potentials in Eq. (11) the experimental $r_{0}$ can be entirely saturated when the cut-off is removed.

Chiral potentials, although exponentially suppressed at large distances develop powerlike singularities at short distances, $R^{2} U(r) \rightarrow-(R / r)^{n}$ with $R$ the typical short distance scale and $n \geq 3$. For these potentials perturbation theory and orthogonality are clearly incompatible if the cut-off is removed (see e.g. Eq. (9)). Non-perturbatively, however, there is no problem. Indeed, the short distance behaviour of the wave function is

$u_{k}(r) \rightarrow C\left(\frac{r}{R}\right)^{n / 4} \sin \left[\frac{2}{n-2}\left(\frac{R}{r}\right)^{\frac{n}{2}-1}+\varphi\right] \quad(r \rightarrow 0)$.

The short distance phase $\varphi$ must be energy and potential independent because of orthogonality. The steps to follow are straightforward. i) For $k=0$ we fix $\alpha_{0}$ to the physical value. ii) We integrate in Eq. (41) and obtain the short distance phase $\varphi_{0}$ from Eq. (10 ) iii) For $k \neq 0$ we use $\varphi_{k}=\varphi_{0}$ (orthogonality constraint from Eq. (6]) ) and iv) We integrate out and obtain $\delta_{0}$ from Eq. (3) . With this procedure we predict $\delta_{0}(k)$ from the potential and the scattering length $\alpha_{0}$ as independent quantities. An outstanding result is the universal low energy theorem for the effective range, where the potential and scattering 
Table 2

Deuteron properties and low energy parameters in the ${ }^{3} S_{1}-{ }^{3} D_{1}$ channel for pionless, $U=0$, the OPE and the TPE potential with $\gamma=\sqrt{2 \mu_{n p} B}$ with $B=2.224575(9)$. Errors in TPE reflect the uncertainties in $\gamma, \eta$ and $\alpha_{0}$ only. NijmII and Reid93 from [1].

\begin{tabular}{|cccccccc|}
\hline & $\gamma\left(\mathrm{fm}^{-1}\right)$ & $\eta$ & $A_{S}\left(\mathrm{fm}^{-1 / 2}\right)$ & $r_{m}(\mathrm{fm})$ & $Q_{d}\left(\mathrm{fm}^{2}\right)$ & $\alpha_{0}(\mathrm{fm})$ & $r_{0}(\mathrm{fm})$ \\
\hline$U=0$ & Input & 0 & 0.6806 & 1.5265 & 0 & 4.3177 & 0 \\
OPE & Input & 0.02633 & 0.8681 & 1.9351 & 0.2762 & 5.335 & 1.638 \\
TPE & Input & Input & $0.884(4)$ & $1.967(6)$ & $0.276(3)$ & Input & $1.76(3)$ \\
\hline \hline NijmII & 0.23160 & 0.02521 & $0.8845(8)$ & 1.9675 & 0.2707 & 5.418 & 1.753 \\
Reid93 & 0.23160 & 0.02514 & $0.8845(8)$ & 1.9686 & 0.2703 & 5.422 & 1.755 \\
\hline Exp. & 0.23160 & $0.0256(4)$ & $0.8846(9)$ & $1.971(6)$ & $0.2859(3)$ & $5.419(7)$ & $1.753(8)$ \\
\hline
\end{tabular}

length dependences can be disentangled analytically, due to the superposition principle,

$r_{0}=2 \int_{0}^{\infty} d r\left(1-u_{0, c}^{2}\right)-\frac{4}{\alpha_{0}} \int_{0}^{\infty} d r\left(r-u_{0, c} u_{0, s}\right)+\frac{2}{\alpha_{0}^{2}} \int_{0}^{\infty} d r\left(r^{2}-u_{0, s}^{2}\right)$,

where $u_{0, c}(r) \rightarrow 1$ and $u_{0, s}(r) \rightarrow r$ for large $r$ are zero energy solutions (see Ref. [11] for more results of this sort and further details). Numerically we find (everything in fm )

$$
\begin{array}{ll}
r_{0}=1.308-\frac{4.548}{\alpha_{0}}+\frac{5.193}{\alpha_{0}^{2}} & (\mathrm{LO}) \\
r_{0}=2.122-\frac{4.889}{\alpha_{0}}+\frac{5.499}{\alpha_{0}^{2}} & (\mathrm{NLO}), \\
r_{0}=2.672-\frac{5.755}{\alpha_{0}}+\frac{6.031}{\alpha_{0}^{2}} & (\mathrm{NNLO})
\end{array}
$$

The cut-off dependence of the effective range can be seen in Fig. 1 for OPE and TPE. As we see in the NNLO case $r_{0}$ nicely approaches the experimental value as the cut-off is removed $r_{c} \rightarrow 0$, supporting the orthogonality constraints and the fact the all contributions of the effective range can be deduced from a good knowledge of the potential in a long distance expansion and model independent fashion. In table 1 we compare results for fully iterated potentials which need only one counterterm with a distorted waves perturbation theory on the leading OPE interaction where there is a proliferation of counterterms without any substantial improvement. The extension to peripheral waves with $j \leq 5$ has been carried out in Ref. [13] for OPE (reproducing [14]) and non-perturbative TPE.

\section{The deuteron}

The analysis for the deuteron equations has been carried out for OPE and TPE in Refs. [ 15] and [11] respectively. Some results are compiled in Table 2] As we see they are rather good as compared with realistic potential models. Actually, at NNLO predictive power is lost, i.e. theoretical predictions have larger error bars than experimental uncertainties. So, there is a lack of motivation for doing $\mathrm{N}^{3} \mathrm{LO}$. Moreover, the chiral constants are determined from ${ }^{1} S_{0},{ }^{3} S_{1}-{ }^{3} D_{1}$ scattering data and the deuteron yielding $c_{1}=-1.2 \pm 0.2$, $c_{3}=-2.6 \pm 0.1$ and $c_{4}=+3.3 \pm 0.1$ (in units of $\mathrm{GeV}^{-1}$ ). 
We have also analyzed $\pi d$ scattering at threshold in the fixed center approximation [ 16. The multiple scattering series yields (recoil and binding are neglected)

$a_{\pi d}=2\left[b_{0}+\left(b_{0}^{2}-2 b_{1}^{2}\right)\left\langle r^{-1}\right\rangle+\left(b_{0}^{3}-2 b_{1}^{2} b_{0}-2 b_{1}^{3}\right)\left\langle r^{-2}\right\rangle+\left(b_{0}^{4}-4 b_{1}^{2} b_{0}^{2}+2 b_{1}^{4}\right)\left\langle r^{-3}\right\rangle+\ldots\right]$

where $b_{0}$ and $b_{1}$ are isoscalar and isovector $\pi N$ scattering lengths . These inverse moments become convergent precisely because the potential is iterated to all orders. The cut-off dependence for $\left\langle r^{-2}\right\rangle$ can be seen in Fig. 1 for OPE and TPE and remarkable agreement for TPE as $r_{c} \rightarrow 0$ with the NijmII results is observed despite the short distance enhancement. These moments diverge in perturbation theory on boundary conditions or distorted OPE waves. $\left\langle r^{-3}\right\rangle$ is convergent for TPE and divergent for Nijm II and Reid93.

\section{Summary}

Perturbative treatments are systematic but require many more counterterms than fully iterated potentials when the UV cut-off is removed. On the other hand, renormalized non-perturbative approaches suffer from a lack of systematics a priori in the sense that strict dimensional power counting does not hold; corrections are parametrically but non-analytically small and the number of counterterms depends crucially on the attractive/repulsive character of the chiral potential at short distances. Within this context we find that Weinberg's power counting is incompatible with renormalization for OPE (confirming [14]) and TPE, perhaps due to an incomplete inclusion of all TPE effects which have similar range. Our approach focuses more on model independent long distance correlations, not necessarily embodied by dimensional power counting. Among those, we find central phases from TPE to be largely explained just in terms of their pure Van der Waals components whose coefficients depend on the chiral constants, suggesting a unexpected connection to the liquid drop model from non-perturbative renormalized chiral dynamics.

\section{REFERENCES}

1. J. J. de Swart, C. P. F. Terheggen and V. G. J. Stoks, arXiv:nucl-th/9509032.

2. P. F. Bedaque and U. van Kolck, Ann. Rev. Nucl. Part. Sci. 52, 339 (2002)

3. E. Epelbaum, Prog. Part. Nucl. Phys. 57 (2006) 654

4. N. Kaiser, R. Brockmann and W. Weise, Nucl. Phys. A 625, 758 (1997)

5. M. C. M. Rentmeester, R. G. E. Timmermans, J. L. Friar and J. J. de Swart, Phys. Rev. Lett. 82, 4992 (1999)

6. D. R. Entem and R. Machleidt, Phys. Rev. C 68, 041001 (2003)

7. E. Epelbaum, W. Glockle and U. G. Meissner, Nucl. Phys. A 747, 362 (2005)

8. M. Pavon Valderrama and E. Ruiz Arriola, Phys. Lett. B 580, 149 (2004)

9. M. Pavon Valderrama and E. Ruiz Arriola, Phys. Rev. C 70, 044006 (2004)

10. M. Pavon Valderrama and E. Ruiz Arriola, arXiv:nucl-th/0410020.

11. M. Pavon Valderrama and E. Ruiz Arriola, arXiv:nucl-th/0506047.

12. D. R. Phillips and T. D. Cohen, Phys. Lett. B 390, 7 (1997)

13. M. Pavon Valderrama and E. Ruiz Arriola, arXiv:nucl-th/0507075.

14. A. Nogga, R. G. E. Timmermans and U. van Kolck, Phys. Rev. C 72 (2005) 054006

15. M. Pavon Valderrama and E. Ruiz Arriola, Phys. Rev. C 72, 054002 (2005) 
16. M. Pavon Valderrama and E. Ruiz Arriola, arXiv:nucl-th/0605078 